\title{
Infinitesimally flexible meshes and discrete minimal surfaces
}

\author{
J. Wallner and H. Pottmann
}

\begin{abstract}
We explore the geometry of isothermic meshes, conical meshes, and asymptotic meshes around the Christoffel dual construction of a discrete minimal surface. We present a discrete Legendre transform which realizes discrete minimal surfaces as conical meshes. Conical meshes turn out to be infinitesimally flexible if and only if their spherical image is isothermic, which implies that discrete minimal surfaces constructed in that way are infinitesimally flexible, and therefore possess reciprocal-parallel meshes. Those are discrete minimal surfaces in their own right. In our study of relative kinematics of infinitesimally flexible meshes we encounter characterizations of flexibility and isothermicity which are of incidence-geometric nature and are related to the classical Desargues configuration. The Lelieuvre formula for asymptotic meshes leads to another characterization of isothermic meshes in the sphere which is based on triangle areas.
\end{abstract}

Key words: conical meshes, discrete minimal surfaces, reciprocal-parallel meshes.

MSC 2000: 53A40, 52C99, 51B15, 65D18

\section{Introduction}

The geometric properties of meshes (i.e., simplicial manifolds with boundary) are an important topic within discrete differential geometry, not only relevant to pure mathematics, but also to geometric modeling and approximation theory [10], computer graphics [9] and building science [11]. These ramifications can for instance be observed for the concept of a conical mesh, which was introduced by [11] with a viewpoint towards architectural design of freeform glass structures, and which fits nicely into the 'consistency as integrability' framework underlying the monograph [5] by A. Bobenko and Yu. Suris.

The present paper contributes to a different subject, namely discrete minimal surfaces, the interplay of properties of meshes like isothermicity and infinitesimal 

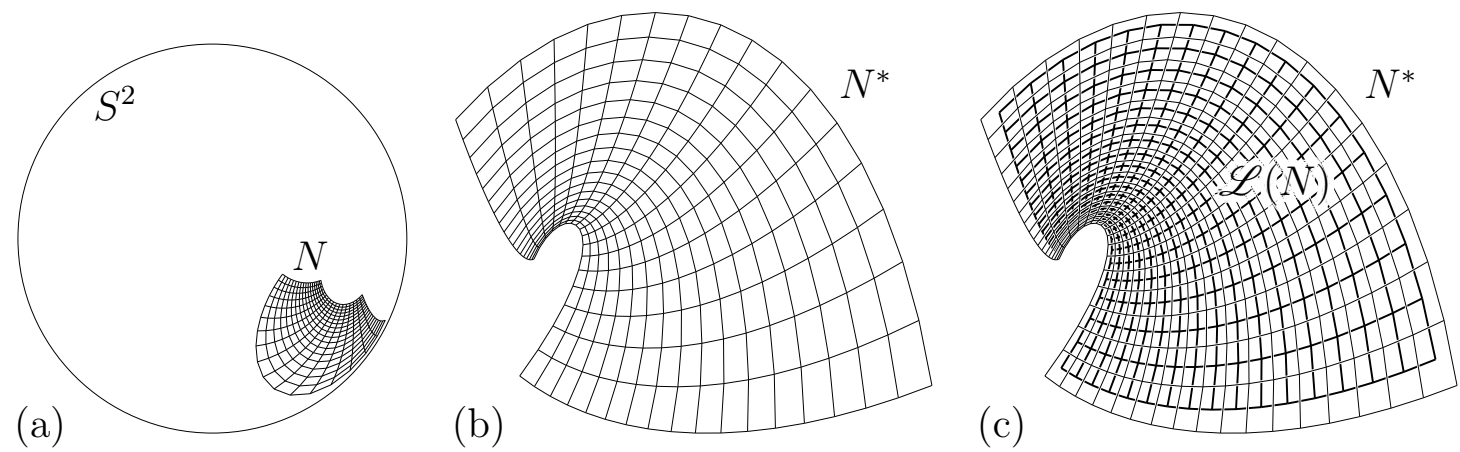

Figure 1: (a) An isothermic mesh $N$ contained in the unit sphere.

(b) The minimal surface $N^{*}=\mathscr{C}(N)$ (Christoffel dual).

(c) $N^{*}$ on top of its Legendre transform $\mathscr{L}(N)$, which is a conical mesh representing the same minimal surface.

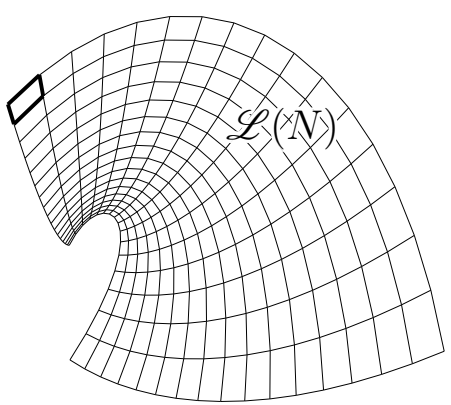

(a)

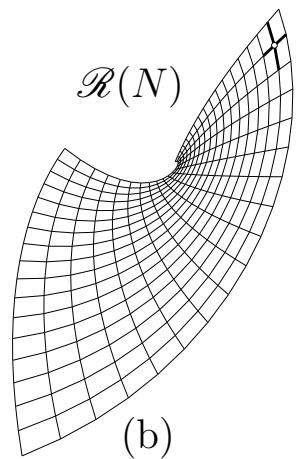

(b)

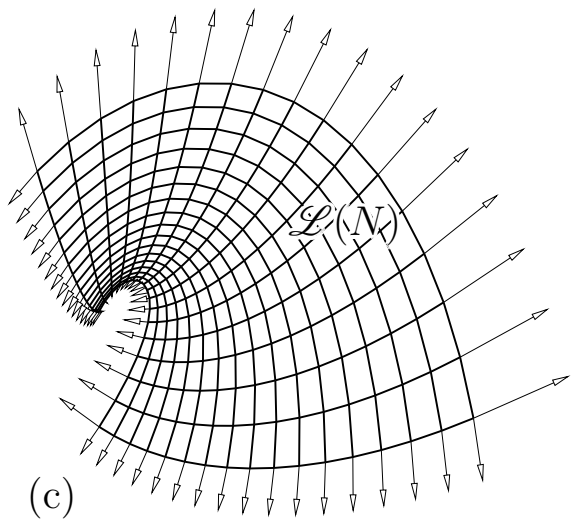

(c)

Figure 2: (a) If the quads of the conical mesh $V$ are considered rigid and joined with their neighbours with hinges, then $V$ is infinitesimally flexible $\Longleftrightarrow V=\mathscr{L}(N)$ as shown by Fig. 1(a)-(c).

(b) A discrete minimal surface $\mathscr{R}(N)$ reciprocal-parallel to $\mathscr{L}(N)$.

(c) $\mathscr{L}(N)$ is in equilibrium with the edges of $\mathscr{R}(N)$ as forces.

flexibility in this context, and the nonlinear incidence geometry of conical and conical-isothermic meshes from the Laguerre geometry viewpoint. There is, however, again a connection to architecture and statics: The edges of a conical mesh in the shape of a discrete minimal surface turn out to be in static equilibrium.

The following subsections gives an overview of previous work on the topics studied by the present paper as well as an outline of the paper. 


\subsection{Previous work}

The notion of discrete minimal surface is not uniquely defined, as the various properties which characterize smooth minimal surfaces can be discretized in different ways, and several of those lead to discrete surfaces with interesting geometric properties. We would like to mention $[13,14,15]$, where area minimizing triangle meshes are studied. For instance, it can be shown that they allow for discrete analogues of conjugate minimal surfaces.

More closely related to the present paper are discretizations of the Christoffel dual construction, which converts an isothermic parametrization of the unit sphere into an isothermic parametrization of a minimal surface, and vice versa. In [3], A. Bobenko, T. Hoffmann and B. Springborn define a Christoffel dual for Schramm circle patterns in the unit sphere, thus succeeding to construct circle and sphere packings in the shape of discrete minimal surfaces. It is remarkable that the geometry of such surfaces can be effectively determined from the mesh combinatorics, which in the smooth case would mean that a minimal surface is computed from the combinatoris of its network of principal curvature lines.

The present study of discrete minimal surfaces is based on the 1996 paper by A. Bobenko and U. Pinkall [4], which introduces a Christoffel dual construction for isothermic meshes in the unit sphere. Isothermic meshes are quadrilateral meshes where all quadrilaterals have a circumcircle, i.e., they are a special case of circular meshes. These meshes, which from the structural viewpoint belong to Möbius geometry, have been introduced by [12]. We refer to the monograph [5] for an overview and further references. The related concept of conical mesh as introduced by $[11,21,17]$ is a Laguerre geometry analogue of a circular mesh. See [7] for a recent unified treatment of circular and conical meshes from the viewpoint of Lie sphere geometry, which establishes the analogies between circular and conical meshes from the viewpoint of the 'consistency as integrability' paradigm [5]. The relation between Moutard nets and isothermic nets is established by [6], which treats topics close to the present paper.

Another property of meshes relevant for our work is infinitesimal flexibility. The relation of this property to the existence of reciprocal-parallel meshes is the topic of the early contributions by R. Sauer $[18,19]$ and the recent paper [20]. The monograph [19] on difference geometry is a source of many of the fundamental properties of meshes which now play a role in discrete differential geometry. A discussion of asymptotic meshes as well as systems of forces in the edges of meshes can also be found there. 


\subsection{Contributions and overview}

The present paper is organized as follows: In Section 2 we review cross ratios, and how the Desargues configuration of projective geometry looks like in Möbius geometry. Section 3, after defining isothermic meshes, gives a characterization of isothermic meshes in the unit sphere in terms of incidence geometry, thereby extending a result of A. Bobenko. Next, Section 3 derives a relation between infinitesimal flexibility of planar quadrilateral meshes and the existence of many Desargues configurations formed by the lines of intersection between face planes of such meshes. We show that a conical mesh is infinitesimally flexible if and only if its spherical image is isothermic. Section 5 deals with the interpretation of conical meshes as the circular meshes of Laguerre geometry, and characterizes infinitesimally flexibility conical meshes in terms of incidence geometry.

The topic of Section 6 is the construction and properties of discrete minimal surfaces. While Section 6.1 reviews the discrete Christoffel dual construction, i.e., discrete minimal surfaces in the shape of circular meshes, Section 6.2 shows how to find a conical mesh which represents the same minimal surface, and which can be seen as a Legendre transform of the Christoffel dual. Section 6.3 discusses meshes which are reciprocal-parallel to conical meshes which represent discrete minimal surfaces in the sense of the previous subsection. Such reciprocal-parallel meshes exist in an essentially unique way and can be regarded as discrete minimal surfaces in the shape of an asymptotic mesh. The so-called Lelieuvre formula for the construction of asymptotic meshes whose spherical image is known is used in Section 6.4 to characterize spherical isothermic meshes in terms of triangle areas which occur in that mesh. Finally, Section 6.5 deals with the relation between reciprocal-parallel meshes and the statics of a mesh: It turns out that for discrete minimal surfaces in the shape of a conical mesh there is an essentially unique collection of nonzero equilibrium forces in its edges.

\section{Geometric Preliminaries}

\subsection{Cross ratios}

We would like to define the cross ratio of 4 points in the real or complex projective lines $\mathbb{R} \cup\{\infty\}$ or $\mathbb{C} \cup\{\infty\}$ by

$$
\operatorname{cr}(a, b, c, d)=(a-b)(b-c)^{-1}(c-d)(d-a)^{-1} .
$$

The cross ratio is a projective invariant, which in the case of $\mathbb{C}$ means that it is a planar Möbius invariant. Co-planar points $a, \ldots, d \in \mathbb{R}^{d}$ can be identified with complex numbers and Equ. (1) is used to define their cross ratio. It is well known 


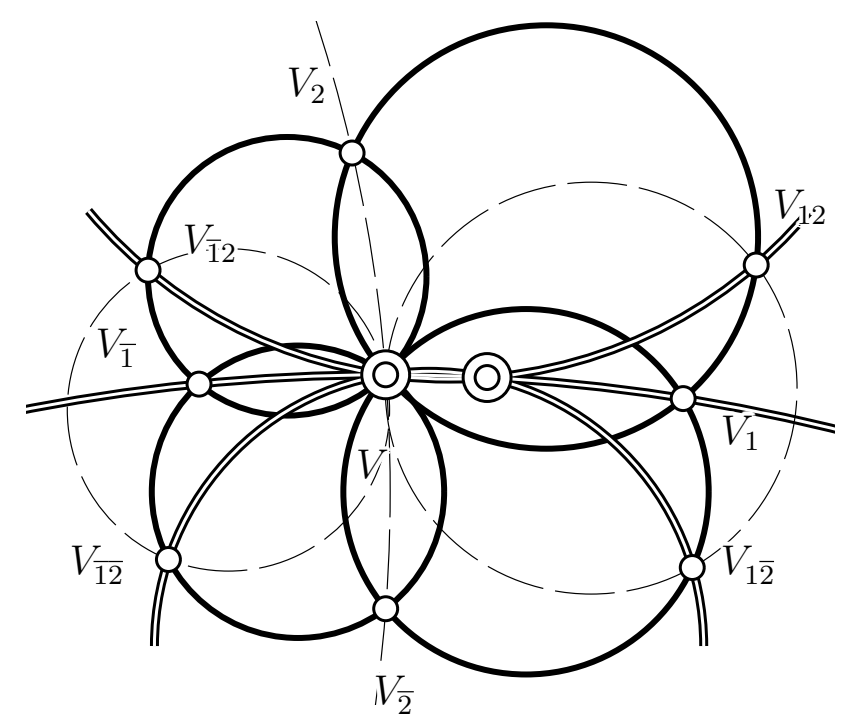

Figure 3: A configuration of coaxial circles which is a Desargues configuration in the Möbius geometry sense.

that then the cross ratio is a Möbius invariant, and that $\operatorname{cr}(a, \ldots, d) \in \mathbb{R} \cup\{\infty\}$ if and only if $a, \ldots, d$ are collinear or cocircular (see also the introduction into cross ratios in [4]).

For points $a, \ldots, d \in \mathbb{R}^{d}$ contained in a conic section $C$, the cross ratio $\operatorname{cr}(a, \ldots$, $d \mid C)$ is defined as $\operatorname{cr}\left(a^{\prime}, \ldots, d^{\prime}\right)$, where $a^{\prime}, \ldots, d^{\prime}$ arise from $a, \ldots, d$ by projection from any center in $C$ onto a straight line. If $C$ is a circle, this cross ratio coincides with the previous one.

\subsection{The Desargues configuration in Möbius geometry}

The Desargues configuration is a basic construction in projective spaces. It is illustrated by Fig. 4. There is a Möbius geometry counterpart of this configuration, which occurs naturally in the context of isothermic meshes and of infinitesimally flexible meshes, and can be converted into the classical configuration by means of an inversion. We first give a definition:

Definition 1. We call three pairwise different circles or straight lines $C_{1}, C_{2}, C_{3}$ coaxial, if one of the following two conditions is satisfied:

(i) $C_{1} \cap C_{2}=\{p, q\}$ with $p \neq q$ and $C_{3}$ passes through both $p$ and $q$; or

(ii) $C_{1} \cap C_{2}=\{p\}$ and $C_{1}, C_{2}, C_{3}$ contain $p$ and are tangent to each other there. In both cases, $p=\infty, q=\infty$ is allowed.

Such circles are illustrated by Fig. 3, where the three dashed circles are coaxial, as well as the three 'double line' circles. Condition (ii) is the limit case of (i) when 
$p, q$ coincide. This definition implies that 3 lines are coaxial either if they have a finite point in common (another intersection point is at infinity), or if they are parallel (they are tangent to each other at infinity).

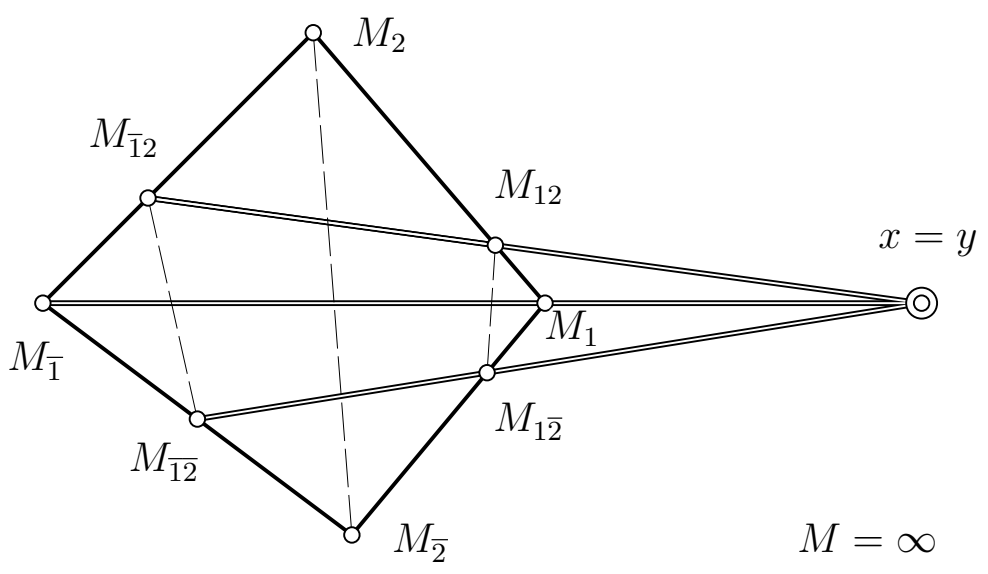

Figure 4: An inversion maps the configuration of Fig. 3 to a classical Desargues configuration. All circles are mapped to straight lines.

The following is an observation of A. Bobenko: Consider the configuration of points $V, V_{\overline{1}}, \ldots, V_{\overline{1} 2}$ such that each of the quadrangles $\left\{V, V_{\overline{1}}, V_{\overline{12}}, V_{\overline{2}}\right\},\left\{V, V_{\overline{2}}, V_{1 \overline{2}}\right.$, $\left.V_{1}\right\},\left\{V, V_{1}, V_{12}, V_{2}\right\},\left\{V, V_{2}, V_{\overline{1} 2}, V_{\overline{1}}\right\}$ has a circumcircle, as illustrated by Fig. 3. An inversion with center $V$ maps $V, \ldots$ to $M, \ldots$, such that the sets $\left\{M_{\overline{1}}, M_{\overline{12}}, M_{\overline{2}}\right\}$, $\left\{M_{\overline{2}}, M_{1 \overline{2}}, M_{1}\right\},\left\{M_{1}, M_{12}, M_{2}\right\},\left\{M_{2}, M_{\overline{1} 2}, M_{\overline{1}}\right\}$ are collinear. This configuration is Desargues in the usual sense, if the lines $M_{\overline{12}} \vee M_{1 \overline{2}}, M_{\overline{1}} \vee M_{1}$, and $M_{12} \vee M_{\overline{12}}$ are concurrent or parallel (i.e., coaxial). By Desargues' theorem coaxiality of these lines is equivalent to the lines $M_{\overline{12}} \vee M_{\overline{1} 2}, M_{\overline{2}} \vee M_{2}, M_{1 \overline{2}} \vee M_{12}$ being coaxial. In the original domain the Desargues property is expressed by the condition that the circumcircles of $\left\{V, V_{\overline{12}}, V_{1 \overline{2}}\right\},\left\{V, V_{\overline{1}}, V_{1}\right\}$, and $\left\{V, V_{12}, V_{\overline{1} 2}\right\}$ are coaxial; and an equivalent condition is that the circumcircles of $\left\{V, V_{\overline{12}}, V_{\overline{1} 2}\right\},\left\{V, V_{\overline{2}}, V_{2}\right\}$, and $\left\{V, V_{1 \overline{2}}, V_{12}\right\}$ are coaxial.

\section{Isothermic meshes}

Discrete isothermic surfaces, or isothermic meshes, have been introduced by [4]. They are quadrilateral meshes all of whose faces are planar and have a circumcircle; in addition the vertices of the mesh must fulfill a cross ratio condition (see below). Isothermic meshes are a discrete analogue of isothermic curvature line parametrizations of smooth surfaces, and are especially useful in the context of 
discrete minimal surfaces. This paper contributes some hitherto unknown properties of isothermic meshes with a focus on meshes in the unit sphere (see Fig. 7).

\subsection{Definitions and notation}

Quadrilateral meshes in general are mappings from $\mathbb{Z}^{2}$ into a space of points. Before we define isothermicity of such a mesh, we explain the subscript notation for index shifts which we will employ later in the paper. We usually denote such a mapping by a capital letter, like $V$. The vertex corresponding to the index pair $(i, j) \in \mathbb{Z}^{2}$ is denoted by $V(i, j)$. Subscripts of the form $V_{k}$ and $V_{\bar{k}}$ denote a right or left index shift in the $k$ th coordinate:

$$
V_{1}(i, j)=V(i+1, j), V_{\overline{2}}(i, j)=V(i, j-1), V_{12}(i, j)=V(i+1, j+1),
$$

and so on. We use the word quadrilateral mesh to indicate a mapping $V$ from $\mathbb{Z}^{2}$ to $\mathbb{R}^{d}$. A planar mesh will be one where $V, V_{1}, V_{2}, V_{12}$ are co-planar throughout the mesh, and the mesh is circular, if these vertices possess a circumcircle. Following [4], for a planar quadrilateral mesh we define the cross ratios

$$
Q=\operatorname{cr}\left(V, V_{1}, V_{12}, V_{2}\right)
$$

The mesh is circular if and only if $Q$ takes values in $\mathbb{R} \cup\{\infty\}$. A circular mesh is defined to be isothermic, if

$$
Q Q_{12}=Q_{1} Q_{2} \quad \text { or } \quad Q: Q_{1}=Q_{2}: Q_{12} .
$$

This is equivalent to the existence of sequences $\alpha: \mathbb{Z} \rightarrow \mathbb{R}$ and $\beta: \mathbb{Z} \rightarrow \mathbb{R}$ with

$$
Q(i, j)=-\beta(j) / \alpha(i)
$$

It is obvious that both circular meshes and isothermic meshes are objects of Möbius geometry.

\subsection{Incidence geometry of isothermic meshes}

It is interesting that the property of being an isothermic mesh can be formulated purely in terms of geometric objects of Möbius geometry and their intersections, without resorting to cross ratios.

Theorem 1. A mesh $V: \mathbb{Z}^{2} \rightarrow \mathbb{R}^{d}$ is isothermic, if and only if each vertex $V(i, j)$ together with its 8 neighbours is aligned in a Desargues configuration in the Möbius sense, i.e., the three circumcircles of $\left\{V_{\overline{1} 2}, V, V_{12}\right\},\left\{V_{\overline{1}}, V, V_{1}\right\},\left\{V_{\overline{12}}, V, V_{1 \overline{2}}\right\}$ are co-axial throughout the mesh (see Fig. 3). 
Proof. An inversion with center $V$ maps the points $V_{1}, \ldots$ to points to $M_{1}, \ldots$ (cf. the discussion in Section 2.2). Consider the intersection point $x=\left(M_{12} \vee M_{\overline{12}}\right) \cap$ $\left(M_{1} \vee M_{\overline{1}}\right)$ and $y=\left(M_{12} \vee M_{\overline{1} 2}\right) \cap\left(M_{1} \vee M_{\overline{1}}\right)$. Coaxiality means that $x=y$. Isothermicity, on the other hand, is expressed in terms of Equation (3) (note the index shift):

$$
\frac{Q}{Q_{\overline{2}}}=\frac{M_{1}-M_{1 \overline{2}}}{M_{1 \overline{2}}-M_{\overline{2}}} \cdot \frac{M_{\overline{2}}-M_{\overline{12}}}{M_{\overline{12}}-M_{\overline{1}}}=\frac{M_{\overline{1}}-M_{\overline{1} 2}}{M_{\overline{1} 2}-M_{2}} \cdot \frac{M_{2}-M_{12}}{M_{12}-M_{1}}=\frac{Q_{\overline{1}}}{Q_{\overline{12}}} .
$$

By the theorem of $\mathrm{G}$. Ceva on the ratios which occur when a line intersects a triangle, these two fractions reduce to $\left(M_{1}-x\right) /\left(x-M_{\overline{1}}\right)$ and $\left(M_{\overline{1}}-y\right) /\left(y-M_{1}\right)$, so isothermicity, like coaxiality, is shown to be equivalent to $x=y$.

Remark 1. The previous result has been first stated by A. Bobenko [2, 6], who suggested the following line of proof: It is known that for generic circular meshes, isothermicity can be characterized by the property that a vertex and its four diagonal neighbours are co-spherical (this characterization works only if the four immediate neighbours are not contained in the sphere common to the diagonal neighbours $\left.V_{12}, V_{12}, V_{\overline{12}}, V_{\overline{12}}\right)$. An inversion whose center is the vertex under consideration produces a configuration like the one of Fig. 4, with $M_{12}, M_{12}, M_{\overline{12} 2}$, $M_{\overline{12}}$ co-planar, whence the Desargues configuration holds. The non-generic case would follow by a limit argument.

\section{Infinitesimally flexible meshes}

\subsection{The Desargues configuration of relative axes}

An infinitesimal motion of a mesh in $\mathbb{R}^{3}$ is a continuous assignment of velocity vectors to each point contained in a face of the mesh, such that for each face $F(i, j)$, this mapping is the restriction to $F(i, j)$ of a mapping of the form

$$
M(i, j): \mathbb{R}^{3} \rightarrow \mathbb{R}^{3}, x \mapsto \omega(i, j) \times x+v(i, j) \quad \text { with } \omega(i, j), v(i, j) \in \mathbb{R}^{3} .
$$

$M(i, j)$ is the velocity vector field of a rigid body motion (i.e., we treat the faces as rigid), and by slight abuse of notation we write $M(i, j) \in \mathfrak{s e}_{3}$. Then $M: \mathbb{Z}^{2} \rightarrow \mathfrak{s e}_{3}$ becomes an $\mathfrak{s e}_{3}$-valued quadrilateral mesh.

Our assumption on continuity means that the relative motions $M, M_{1}$ associated with neighbouring faces $F, F_{1}$ assign the same velocity vector to points of the common edge, so $M-M_{1}$ is an infinitesimal rotation about that edge. The same is true for $M-M_{2}$. The edges are thus considered as hinges between rigid faces.

For $M \in \mathfrak{s e}_{3}$, we use the notation $\mathscr{A}(M)$ for its axis, i.e., $x \in \mathscr{A}(M) \Longleftrightarrow$ $M(x)=0$. The axis is a straight line if and only if $M$ is an infinitesimal rotation. 


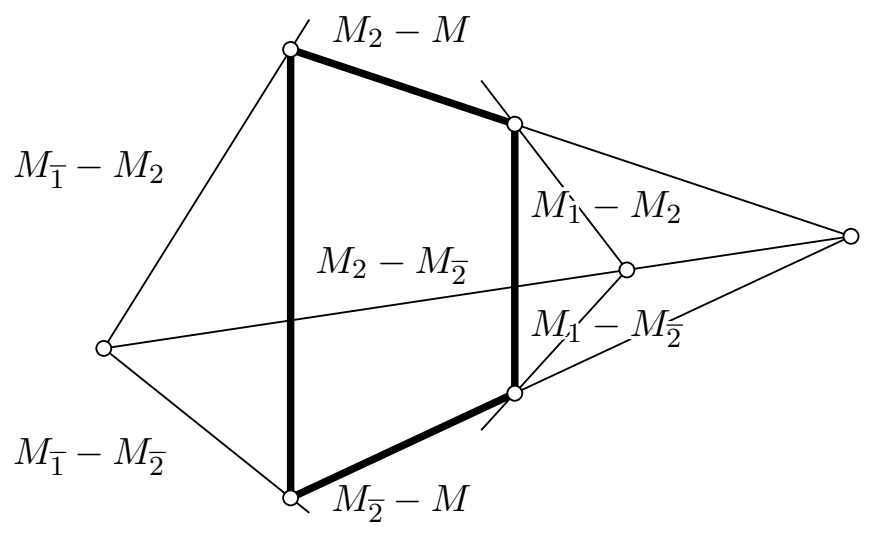

Figure 5: The configuration of relative axes in an infinitesimally flexible mesh. The relative motions $M_{1}-M, \ldots$ are indicated via their axes.

A mesh is infinitesimally flexible, if there exists an assignment $M: \mathbb{Z}^{2} \rightarrow \mathfrak{s e}_{3}$ of an infinitesimal motion to each face, such that the conditions above are fulfilled, but $M$ is not the constant function, i.e., we exclude the case that the entire mesh is moved like a rigid body.

Proposition 1. If $M, M_{1}, M_{2}, M_{\overline{1}}, M_{\overline{2}}$ are infinitesimal motions of the face $F$ and its neighbours, then the axes of the relative motions between them constitute a Desargues configuration as shown by Fig. 4 and, more importantly, Fig. 5. Conversely, given such axes, there exist infinitesimal motions with these relative axes.

This is well known and easy to show [19]. The proof relies on the following fact: If infinitesimal motions $M, M^{\prime}, M^{\prime \prime} \in \mathfrak{s e}(3)$ have the property that both $M-M^{\prime}$

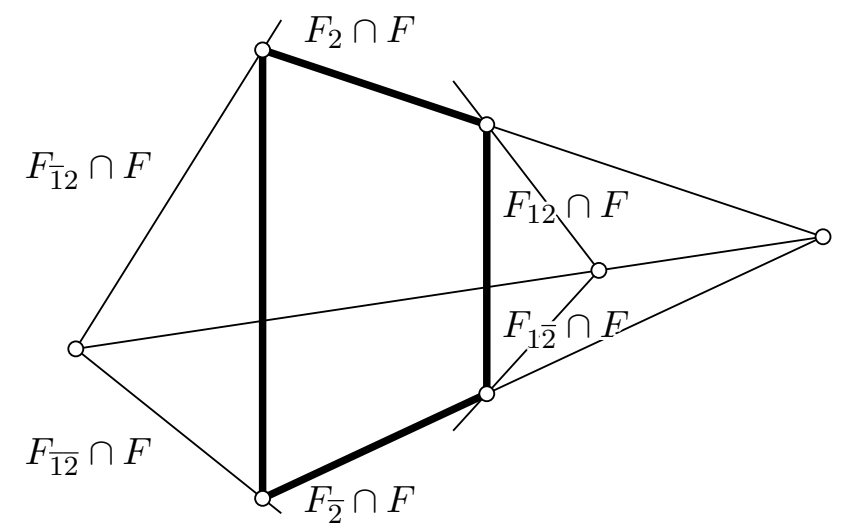

Figure 6: The intersection lines of faces constitute a Desargues configuration in the case of infinitesimal flexibility. 
and $M-M^{\prime \prime}$ are rotations, and the axes intersect, then also $M^{\prime}-M^{\prime \prime}$ is a rotation whose axis passes through the same intersection point (which is assigned the same velocity by all three infinitesimal motions).

Proposition 2. Consider a quadrilateral mesh with planar faces $F(i, j)$. Let $N(F)$ denote the set of 8 neighbour faces of the face $F$. Then the mesh is infinitesimally flexible if and only if for each face, the lines $F \cap F^{\prime}$ for $F^{\prime} \in N(F)$ together with $F_{1} \cap F_{\overline{1}}$ constitute a Desargues configuration according to Fig. 6 . Here we assume that all intersection lines are well defined (alternatively, $F_{2} \cap F_{\overline{2}}$ can be used).

Proof. As to notation, we consider faces $F, F_{1}, \ldots$, corresponding infinitesimal motions $M, M_{1}, \ldots \in \mathfrak{s e}(3)$ and vectors $\omega, \omega_{1}, \ldots$ of angular velocity, which are parallel to the axes $\mathscr{A}(M), \mathscr{A}\left(M_{1}\right), \ldots$, respectively.

Because of $\omega_{1}-\omega_{2}=\left(\omega_{1}-\omega_{12}\right)-\left(\omega_{2}-\omega_{12}\right)$, the axis $\mathscr{A}\left(M_{1}-M_{2}\right)$ lies in the plane spanned by the edges $\mathscr{A}\left(M_{1}-M_{12}\right)$ and $\mathscr{A}\left(M_{2}-M_{12}\right)$. This plane is the face $F_{12}$. Analogously, the axis $\mathscr{A}\left(M_{1}-M_{2}\right)$ is contained in the face $F$ (replace $\omega_{12}$ by $\left.\omega\right)$. It follows that

$$
\mathscr{A}\left(M_{1}-M_{2}\right)=F \cap F_{12} .
$$

With similar arguments we establish the identity of the lines in Fig. 5 (axes) with the corresponding lines of Fig. 6 (intersection lines). Now the 'only if' part of the statement follows directly from Proposition 1.

To show the 'if' part, we use Proposition 1 to construct nontrivial infinitesimal motions $M, M_{1}, M_{2}, M_{\overline{1}}, M_{\overline{2}}$, whose existence follows from the fact that the given axes (i.e., intersection lines) constitute a Desargues configuration. Without loss of generality $M=0$. We write $\omega_{1}-\omega_{2}=\lambda a-\mu b$, where $a, b \in \mathbb{R}^{3}$ are parallel to the edges $F_{12} \cap F_{1}$ and $F_{12} \cap F_{2}$, respectively. Then we let $\omega_{12}:=\omega_{1}-\lambda a=\omega_{2}-\mu b$. With the axis and the vector of angular velocity the relative motion $M_{12}$ is defined. We check for consistency: $\omega_{1}-\omega_{12}=\lambda a$, so $\mathscr{A}\left(M_{12}-M_{1}\right)=F_{12} \cap F_{1}$, and indeed the relative axis $\mathscr{A}\left(M_{12}-M_{1}\right)$ is in its place as required. A similar argument shows consistency with $M_{2}$. The construction of the remaining infinitesimal motions $M_{1 \overline{2}}$, $M_{\overline{12}}, M_{\overline{12}}$ is analogous to the construction of $M_{12}$.

We have thus shown how to construct infinitesimal motions for every $3 \times 3$ piece of the mesh separately. According to [20], this is sufficient for infinitesimal flexibility.

\subsection{Characterization of isothermicity via flexibility}

Consider a mesh with planar faces $F(i, j)$. It can be consistently oriented, so a field of unit normal vectors $N(i, j) \in S^{2}$ is well defined. We call the mesh $N$ 
the spherical image of the first mesh and denote it by " $\mathscr{S}(F)$ ". In case $\mathscr{S}(F)$ is circular, the original mesh is conical [11]. In case $F(i, j)$ is the tangent plane of $S^{2}$ in the point $N(i, j)$, the conical mesh is the polar dual of the spherical circular mesh $N$.

One reason why we are intersed in infinitesimally flexible meshes is that among the conical meshes, the infinitesimally flexible ones have interesting properties related to isothermicity.

Theorem 2. Consider the conical mesh $F$ and its spherical image $N=\mathscr{S}(F)$. Provided the vertices $N, N_{12}, \ldots$ which cocur in (ii) are distinct, the following three properties are equivalent:

(i) $N$ is isothermic;

(ii) Throughout the mesh, the planes $N_{\overline{1} 2} \vee N \vee N_{12}, N_{\overline{1}} \vee N \vee N_{1}, N_{\overline{12}} \vee N \vee N_{1 \overline{2}}$ have a line in common;

(iii) $F$ is infinitesimally flexible.

Proof. The equivalence (i) $\Longleftrightarrow$ (ii) is Theorem 1. In order to show (ii) $\Longleftrightarrow$ (iii) we note that infinitesimal flexibility is a property of $N$ alone [20], so without loss of generality we assume that $F$ is the polar dual of $N$. The Desargues condition of Proposition 2, which is illustrated by Figures 5 and 6 , is by polarity turned into condition (ii).

\section{$5 \quad$ Laguerre-isothermic meshes}

For some constructions with meshes, the basic elements are the planes. It therefore is natural to consider them from the viewpoint of Laguerre geometry, which is the Euclidean geometry of planes. For an introduction into Laguerre geometry and the Blaschke cylinder see the monograp s $[1,8]$, for applications in geometric modeling see $[16]$.

\subsection{The Blaschke cylinder model: definitions and notation}

We describe the Blaschke cylinder as a point model for the oriented planes of Euclidean 3-space. The oriented plane with equation $n_{0}+\langle n, x\rangle=0$ with $n_{0} \in \mathbb{R}$ has the normal vector $n=\left(n_{1}, n_{2}, n_{3}\right) \in S^{2}$ and is represented by the coordinate vector $\left(n_{0}, \ldots, n_{3}\right) \in Z$, where $Z=\mathbb{R} \times S^{2}$ is the Blaschke cylinder.

Definition 2. We consider the projection $\pi:\left(x_{0}, x_{1}, x_{2}, x_{3}\right) \mapsto\left(x_{1}, x_{2}, x_{3}\right)$ and call an affine subspace $U$ of $\mathbb{R}^{4}$ proper if $\pi \mid U$ is $1-1$. If $U$ is proper, then we call $U \cap Z$ an L-sphere in the case $\operatorname{dim} U=3$, and an L-circle if $\operatorname{dim} U=2$. 
The oriented planes tangent to the oriented sphere with center $m$ and radius $r$ fulfill the equation $n_{0}-r+\langle n, m\rangle=0$. Obviously such a set of planes corresponds to an L-sphere and vice versa. If $C=U \cap Z$ is an L-circle, then $\pi(C)$ is a circle in $S^{2}$. An L-circle has always the form $Z \cap U_{1} \cap U_{2}$, where $Z \cap U_{1}$ and $Z \cap U_{2}$ are L-spheres, so an L-circle consists of the planes common to two L-spheres. It may happen that the radii of these spheres are equal, in which case we call the L-circle cylindrical, and in case of zero radius, axial. If the radii are different, then the L-circle will be called conical. It is elementary that the tangent planes of a cone of revolution, of a cylinder of revolution, or a straight line correspond to conical/cylindrical/axial L-circles.

Definition 3. For L-cocircular points $a, b, c, d$, we define a cross ratio via L-cr $(a$, $b, c, d)=\operatorname{cr}(a, b, c, d \mid C)$, where $C$ is the L-circumcircle of the points $a, b, c, d$.

The cross ratio of L-cocircular points is a Laguerre invariant, because Laguerre transformations are projective automorphisms of the Blaschke cylinder, which leave the cross ratio of 4 points on a conic invariant.

\subsection{Conical meshes as L-circular meshes}

Conical meshes as defined in [11] are quadrilateral meshes with planar faces such that for each vertex, the adjacent planes are tangent to a cone of revolution. These planes can be consistently oriented via the mesh's combinatorics, so a conical mesh with faces $F(i, j)$ has the property that throughout the mesh, $F, F_{1}, F_{2}, F_{12}$ are contained in a conical L-circle. Obviously this is also a characterization of conical meshes.

It is tempting to define a conical mesh by the requirement that throughout the mesh the faces $F, F_{1}, F_{2}, F_{12}$ have a conical L-circumcircle. Unfortunately, the property of being conical, or cylindrical, or axial, is not Laguerre invariant. There is however a subgroup of the full Laguerre transformation group which leaves the distinction between conical and non-conical L-circles invariant: This subgroup contains the Euclidean congruence transformations as well as the offsetting operation, which moves each plane by a fixed distance in the direction of its normal vector:

$$
\left(n_{0}, n_{1}, n_{2}, n_{3}\right) \mapsto\left(n_{0}+d, n_{1}, n_{2}, n_{3}\right) .
$$

So there is a slight distinction between L-circular meshes and conical meshes: Every conical mesh is L-circular, but the L-circular meshes have conical vertices together with vertices at infinity where the faces are tangent to a common cylinder or straight line.

Definition 4. A mesh with faces $\widetilde{N}$ is L-isothermic, if the mesh $N=\pi(\widetilde{N})$ of normal vectors is isothermic. 
Note that the symbols $\pi$ (for projection) and $\mathscr{S}$ (for spherical image) mean essentially the same thing.

If the mesh $\pi(\widetilde{N})$ is isothermic, then it is circular. Thus, $\widetilde{N}$ is an L-circular mesh, which implies that it is a conical mesh (if we assume that $\widetilde{N} \operatorname{did}$ not have vertices at infinity from the beginning). By Theorem 2, L-isothermicity means infinitesimal flexibility.

\subsection{Incidence geometry of flexible conical meshes}

We are going to derive a property of L-isothermic meshes, which in the generic case is also a characterization of isothermicity. Similar to the case of isothermic meshes in the unit sphere, we aim at a characterization purely in terms of incidence. A related result which characterizes isothermicity of quadrilateral meshes by the condition that a vertex which is co-spherical with its four diagonal neighbours is shown in [6].

Proposition 3. Consider a conical mesh with faces $F(i, j)$ and its spherical image $\mathscr{S}(F)=N$. If $N$ is isothermic (i.e., $F$ is infinitesimally flexible), then the faces $F, F_{12}, F_{1 \overline{2}}, F_{\overline{12}}, F_{\overline{1} 2}$, are tangent to a common sphere $S$. If at least one of $F_{1}, F_{2}$, $F_{\overline{1}}, F_{\overline{2}}$ is not tangent to $S$, then this condition characterizes isothermicity of $N$.

Here we assume that circumcircles of neighbouring quads of $N$ are distinct.

Proof. Consider the mesh $\widetilde{N}$ in the Blaschke cylinder associated with $F$. The sets $\left\{\widetilde{N}_{\overline{12}}, \widetilde{N}_{\overline{1}}, \widetilde{N}_{\overline{2}}, \widetilde{N}\right\}$ and $\left\{\widetilde{N}_{\overline{1}}, \widetilde{N}_{\overline{1} 2}, \widetilde{N}, \widetilde{N}_{2}\right\}$ are co-planar, so their union is contained in a three-space $U$. $U$ is proper because $\operatorname{dim} \pi(U)<3$ implies that there is only 1 circumcircle of the points under consideration. Analogously, there is a proper 3-space $U^{\prime}$ which contains $\left\{\widetilde{N}_{1 \overline{2}}, \widetilde{N}_{1}, \widetilde{N}_{\overline{2}}, \widetilde{N}, \widetilde{N}_{12}, \widetilde{N}_{2}\right\}$.

Isothermicity of $N$ means that the circumcircles according to Theorem 1 are coaxial. As both $\pi \mid U$ and $\pi \mid U^{\prime}$ are 1-1, the L-circumcircles of the sets $\left\{\widetilde{N}_{\overline{1} 2}, \widetilde{N}, \widetilde{N}_{12}\right\}$ $\left\{\widetilde{N}_{\overline{1}}, \widetilde{N}, \widetilde{N}_{1}\right\}\left\{\widetilde{N}_{\overline{12}}, \widetilde{N}, \widetilde{N}_{1 \overline{2}}\right\}$ either intersect in a second point besides $N$, or they touch each other in $N$. In particular, the planes $\widetilde{N}_{\overline{1} 2} \vee \widetilde{N} \vee \widetilde{N}_{12}$ and $\widetilde{N}_{\overline{12}} \vee \widetilde{N} \vee \widetilde{N}_{1 \overline{2}}$ have a line in common, and consequently the affine span of $\left\{\widetilde{N}, \widetilde{N}_{12}, \widetilde{N}_{1 \overline{2}}, \widetilde{N}_{\overline{12}}, \widetilde{N}_{\overline{1} 2},\right\}$ is a 3 -space (it is proper because otherwise $Z \cap U$ would project to 1 circle). It follows that the corresponding planes are co-spherical as desired.

In order to show the converse, we use $N$ as center of a projection onto any complementary 3-space. The images of points $N_{1}, \ldots$ are denoted by $M_{1}, \ldots$ By assumption, $M_{\overline{12}}, M_{\overline{1} 2}, M_{1 \overline{2}}, M_{12}$ are contained in a plane $\epsilon$, whereas at least one of $M_{1}, M_{2}, M_{\overline{1}}, M_{\overline{2}}$ is not. L-circumcircles are by construction mapped to straight lines, so each of $\left\{M_{\overline{12}}, M_{\overline{1}}, M_{\overline{2}}\right\},\left\{M_{\overline{1}}, M_{\overline{1} 2}, M_{2}\right\},\left\{M_{2}, M_{12}, M_{1}\right\}$, and $\left\{M_{\overline{2}}, M_{1 \overline{2}}, M_{1}\right\}$ is collinear. It follows that none of $M_{\overline{1}}, M_{\overline{2}}, M_{1}, M_{2}$ is contained in $\epsilon$ (if 2 points of a collinear set are contained in a plane, so is the entire set). It follows that we have a non-planar Desargues configuration according to Fig. 4. 
We define cross ratios $\widetilde{Q}=\operatorname{cr}\left(M, M_{1}, M_{12}, M_{2}\right), \widetilde{Q}=\operatorname{L-cr}\left(\widetilde{N}, \widetilde{N}_{1}, \widetilde{N}_{12}, \widetilde{N}_{2}\right)$, and $Q=\operatorname{cr}\left(N, N_{1}, N_{12}, N_{2}\right)$. The same arguments as in the proof of Theorem 1 show that $\widetilde{\widetilde{Q}} \widetilde{\widetilde{Q}}_{12}=\widetilde{\widetilde{Q}}_{1} \widetilde{\widetilde{Q}}_{2}$. Projection of a line onto a conic leaves cross ratios invariant, if the projection center is on that conic, so $\widetilde{Q} \widetilde{Q}_{12}=\widetilde{Q}_{1} \widetilde{Q}_{2}$. Finally the projection $\pi$ leaves cross ratios on conics invariant, so $Q Q_{12}=Q_{1} Q_{2}$. This is what we wanted to show.

\section{Construction of discrete minimal surfaces by dualities}

We briefly review the construction of a discrete minimal surface from a spherical isothermic mesh via the discrete Christoffel duality [4] and then derive two new ones. It should be mentioned that there is at least one further construction of discrete minimal surfaces based on a different definition of discrete isothermic surface, namely the one in [3].

\subsection{Circular meshes - the Christoffel dual construction}

Consider an isothermic mesh $V$ and the sequences $\beta_{j}$ and $\alpha_{i}$ according to Equ. (4). Then the Christoffel dual $V^{*}=\mathscr{C}(V)$ is any isothermic mesh with

$$
V_{1}^{*}-V^{*}=\frac{V_{1}-V}{\alpha\left\|V_{1}-V\right\|^{2}}, \quad V_{2}^{*}-V^{*}=\frac{V-V_{2}}{\beta\left\|V-V_{2}\right\|^{2}} .
$$

$\mathscr{C} \mathscr{C}(V)$ is a scaled copy of $V$ (see [4], which has a typo in the equation corresponding to our Equation (5)). That paper discusses why $N^{*}$ is a discrete Weierstrass representation of a minimal surface, if $N$ is an isothermic mesh in $S^{2}$ such that the cross ratios $Q(i, j)$ of $(2)$ equal -1 . If $Q(i, j) \neq-1$ but rather is given by Equ. (4), then the mesh $N^{*}$ is still a discrete minimal surface: If $g(u+i v)$ is the Weierstrass representation (i.e., an isothermic curvature line parametrization), then such a Christoffel dual corresponds to a surface of the form $g(\phi(u)+i \psi(v))$, where $\phi$ and $\psi$ are parameter transforms.

\subsection{Conical meshes - the Legendre transform of the Chri- stoffel dual}

If $N: \mathbb{Z}^{2} \rightarrow S^{2}$ is isothermic and $N^{*}$ is its Christoffel dual, we consider $N(i, j)$ as normal vector in the point $N^{*}(i, j)$ and define the tangent plane $F(i, j)$ accordingly. The oriented distance of a point from such a tangent plane is defined by $\operatorname{dist}(x, F)=\left\langle x-N^{*}, N\right\rangle$. 


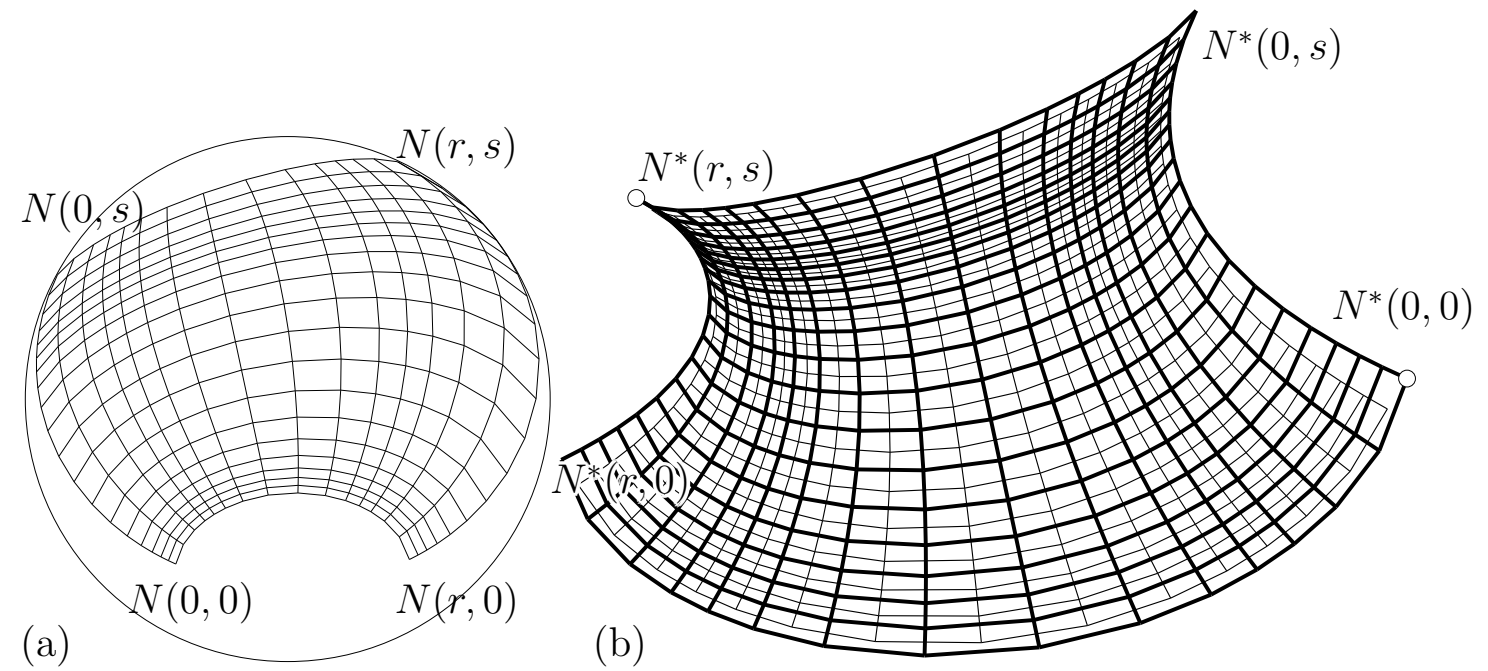

Figure 7: (a) An isothermic mesh $N: \mathbb{Z}^{2} \rightarrow S^{2}$. (b) Corresponding Christoffel dual $N^{*}=\mathscr{C}(N)$ (in bold) and associated conical mesh $\mathscr{L}(N)$. Both meshes $\mathscr{C}(N)$ and $\mathscr{L}(N)$ describe the same underlying minimal surface.

Proposition 4. Assume that $N: \mathbb{Z}^{2} \rightarrow S^{2}$ is isothermic with sequences $\alpha_{i}$ and $\beta_{j}$ according to Equ. (4). Then the tangent planes $F(i, j)$ of the Christoffel dual $N^{*}$ have the property

$$
\operatorname{dist}\left(N^{*}, F_{1}\right)=\operatorname{dist}\left(N_{1}^{*}, F\right)=\frac{1}{2 \alpha}, \operatorname{dist}\left(N^{*}, F_{2}\right)=\operatorname{dist}\left(N_{2}^{*}, F\right)=\frac{-1}{2 \beta} .
$$

The tangent planes $F, F_{1}, F_{12}, F_{2}$ intersect in a common point $V$ on the axis $A$ of the circumcircle of the vertices $N^{*}, N_{1}^{*}, N_{12}^{*}, N_{2}^{*}$.

Proof. We expand the distance of the neighbour vertex $N_{1}^{*}$ from the tangent plane $F$, which has the equation $\left\langle N, x-N^{*}\right\rangle=0$ :

$$
\left\langle N, N_{1}^{*}-N^{*}\right\rangle=\left\langle N, \frac{N_{1}-N}{\alpha\left\|N-N_{1}\right\|^{2}}\right\rangle=\frac{1}{\alpha} \frac{\langle N, N\rangle-\left\langle N, N_{1}\right\rangle}{\langle N, N\rangle-2\left\langle N, N_{1}\right\rangle+\left\langle N_{1}, N_{1}\right\rangle}
$$

which results in $1 / 2 \alpha$, because $\|N\|=\left\|N_{1}\right\|=1$. The computation for the other expression in (6) is similar. Now consider the symmetry plane $S$ of the points $N^{*}$ and $N_{1}^{*}$, whose normal vector is alternatively $N^{*}-N_{1}^{*}$ or $N-N_{1}$. The planes $F, F_{1}, S$ have the equations

$$
\left\langle N, N^{*}-x\right\rangle=0,\left\langle N_{1}, N_{1}^{*}-x\right\rangle=0,\left\langle N-N_{1}, \frac{N^{*}+N_{1}^{*}}{2}-x\right\rangle=0 .
$$

If we observe (6), we see that the difference of the former two equations equals the latter. This implies $F \cap F_{1} \subset S$ and consequently $F \cap F_{1} \cap A \neq\{\}$. Similar 
arguments show that also $F_{1} \cap F_{12}, F_{12} \cap F_{2}, F_{2} \cap F$ meet $A$. These four intersection points obviously coincide.

For the next result, recall that a Combescure transform of a mesh $V$ is another mesh $V^{\prime}$ such that $V-V_{1} \| V^{\prime}-V_{1}^{\prime}$ and $V-V_{2} \| V-V_{2}^{\prime}$ (i.e., corresponding edges are parallel).

Theorem 3. If $N: \mathbb{Z}^{2} \rightarrow S^{2}$ is isothermic, then the tangent planes $F(i, j)$ of the Christoffel dual $N^{*}$ constitute a conical mesh $\mathscr{L}(N)$, whose vertices are situated on the axes of circumcircles of quads in $N^{*}$. Every infinitesimally flexible conical mesh has a Combescure transform of the form $\mathscr{L}(N)$.

Proof. By Proposition 4, the tangent planes $F(i, j)$ are the faces of a mesh, called $\mathscr{L}(N)$, with vertices as stated. $\mathscr{L}(N)$ is conical, because $\mathscr{S}(F)=N$ is circular.

An arbitrary infinitesimally flexible conical mesh $\widetilde{F}$ has an isothermic spherical image $N=\mathscr{S}(\widetilde{F})$. Because of $N=\mathscr{S} \mathscr{L}(N), \widetilde{F}$ and $\mathscr{L}(N)$ have parallel faces and edges.

The conical mesh referred to in Theorem 3 is a discrete minimal surface in the same sense as the Christoffel dual was. It is called a Legendre transform of the Christoffel dual, because in general Legendre transform means the passage from points to tangent planes.

This construction of a conical mesh which represents tangent planes of a circular mesh yields two meshes which describe the same underlying surface. For conical meshes related to circular meshes, such that the faces of the former contain the vertices of the latter, the interested reader is referred to $[17,7]$.

\subsection{Asymptotic and reciprocal-parallel meshes}

Recall that a mesh whose vertices correspond to the faces of a given mesh is combinatorially dual to the given mesh, and that there is a natural bijection of edges (defined by adjacent faces in the given mesh, and by adjacent vertices in the dual mesh). A mesh $V$ is reciprocal-parallel to another mesh $V^{\prime}$, if it is a combinatorial dual and corresponding edges are parallel. We use the notation

$$
V, V^{\prime} \text { are reciprocal-parallel : } \quad V \sim V^{\prime} .
$$

If $V$ has planar faces, $V^{\prime}$ has planar vertex stars, i.e., the edges emanating from a vertex are co-planar. This property characterizes asymptotic meshes (A-meshes). Such meshes are a discretization of the network of asymptotic curves [19]. A reciprocal-parallel mesh, if it exists, is unique up to scaling, if never more than two edges emanating from a vertex of the given mesh are co-planar. For details, see $[19,20]$. The vertices $N(i, j)$ of the spherical image mesh $N=\mathscr{S}(V)$ are the normal vectors associated with the vertices $V(i, j)$. 


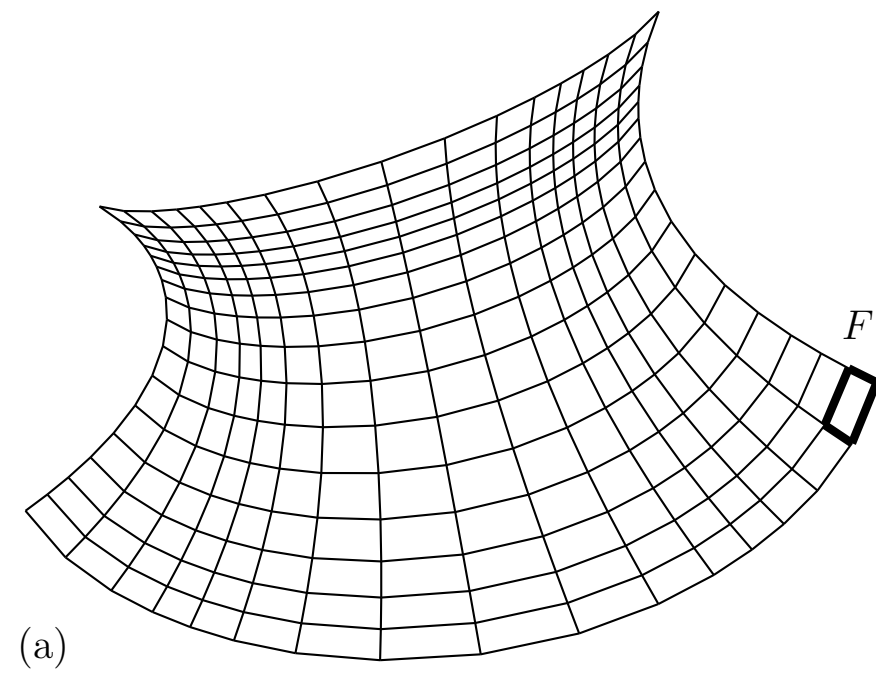

(b) $\quad V(1,1)$

Figure 8: (a) A discrete minimal surface of the form $\mathscr{L}(N)$ with faces $F(i, j)$ the same as in Fig. 7). (b) The corresponding reciprocal-parallel mesh $V=\mathscr{R}(N)$.

Theorem 4. We assume that $F$ is a conical mesh with spherical image $N$, and that for the mesh $N$, the four vertices in each quad are distinct. Then the following are equivalent

(i) F is infinitesimally flexible;

(ii) F possesses a reciprocal-parallel mesh, which then is asymptotic;

(iii) $N$ is isothermic.

Proof. The first equivalence in is well known [19, 20], and so the theorem follows from Theorem 2.

The asymptotic mesh which is reciprocal-parallel to the mesh $F$ in Theorem 4 is uniquely determined, up to scale, by the spherical image $N$ alone [19, 20]. We therefore employ the notation $\mathscr{R}(N)$ for such meshes.

Theorem 5. The circular mesh $N: \mathbb{Z}^{2} \rightarrow S^{2}$ is the spherical image of an asymptotic mesh $V=\mathscr{R}(N)$ if and only if $N$ is isothermic (we assume that the four vertices of every quad in $N$ are distinct).

Proof. We argue as follows: If $N=\mathscr{S}(V)$, then $V \sim \mathscr{L}(N)$ by construction, and by Theorem $4, N$ is isothermic. If $N$ is isothermic, $\mathscr{L}(N)$ is infinitesimally flexible and Theorem 4 shows that there exists a reciprocal-parallel mesh. 
Definition 5. If $N: \mathbb{Z}^{2} \rightarrow S^{2}$ is isothermic so that $\mathscr{R}(N)$ exists, we call $\mathscr{R}(N)$ a minimal A-mesh.

The reasons why we regard $\mathscr{R}(N)$ as a discrete minimal surface is that it is an asymptotic mesh whose spherical image is isothermic. The smooth variant of this property is that asymptotic curves of a surface have orthogonal spherical image curves. This characterizes minimal surfaces.

We can prescribe two polygons of the spherical image $N$ and construct a minimal A-mesh from it (because $N$ is determined by these data, and in turn $\mathscr{R}(N)$ is determined up to scale). As already mentioned by [4], solving this Cauchy problem is quite unstable numerically. We however did not experience numerical instabilities when constructing the reciprocal mesh from any of the many known discrete minimal surfaces in the sense of [4].

At this point we would like to summarize the connections between various types of meshes and results we have obtained so far:

- If a mesh $N: \mathbb{Z}^{2} \rightarrow S^{2}$ is isothermic, then its polar dual is infinitesimally flexible, and vice versa.

- If a mesh $N: \mathbb{Z}^{2} \rightarrow S^{2}$ is isothermic, then the meshes $\mathscr{C}(N)$ (circular) and $\mathscr{L}(N)$ (conical) are Legendre transforms of each other and represent the same minimal surface.

- If a mesh $N: \mathbb{Z}^{2} \rightarrow S^{2}$ is isothermic, there exists an asymptotic mesh $\mathscr{R}(N)$, whose spherical image is $N$, and vice versa.

- For any spherical isothermic mesh $N$, its polar dual and $\mathscr{L}(N)$ are Combescure transforms of each other. $\mathscr{R}(N)$ is reciprocal-parallel to both of them.

\subsection{The Lelieuvre formula and area properties of isother- mic meshes}

The existence of an asymptotic mesh $V$ with given spherical image $N=\mathscr{S}(V)$ is equivalent to the existence of normal vectors $\widetilde{N}(i, j)=\lambda(i, j) N(i, j)$ such that

$$
\left\{\widetilde{N}+\widetilde{N}_{12}, \widetilde{N}_{1}+\widetilde{N}_{2}\right\} \text { linearly dependent. }
$$

(see e.g. [20]). In that case, apart from a scaling factor, $V$ is given by $V-V_{1}=$ $\widetilde{N} \times \widetilde{N}_{1}$ and $V_{2}-V=\widetilde{N} \times \widetilde{N}_{2}$. This is called the Lelieuvre formula. We use this fact to derive a statement about triangle areas in spherical isothermic meshes.

Proposition 5. A circular mesh $N: \mathbb{Z}^{2} \rightarrow S^{2}$ is isothermic, if and only if the following condition regarding the areas " $\Delta(a, b, c)$ " of triangles holds true:

$$
\frac{\Delta\left(N N_{1} N_{12}\right) \Delta\left(N N_{2} N_{\overline{1} 2}\right) \Delta\left(N N_{\overline{1}} N_{\overline{12}}\right) \Delta\left(N N_{\overline{2}} N_{1 \overline{2}}\right)}{\Delta\left(N N_{12} N_{2}\right) \Delta\left(N N_{\overline{1} 2} N_{\overline{1}}\right) \Delta\left(N N_{\overline{12}} N_{\overline{2}}\right) \Delta\left(N N_{1 \overline{2}} N_{1}\right)}=1,
$$


i.e., the product of areas of the four shaded triangles in Fig. 9b equals the product of areas of the other four triangles.

Proof. By Theorem 5, $N$ is isothermic if and only if there is an asymptotic mesh $V$ with $N=\mathscr{S}(V)$, i.e., if and only if there is $\widetilde{N}=\lambda N$ such that (8) is fulfilled. From the Grassmann identity

$$
\begin{aligned}
\left(N \times N_{12}\right) \times\left(N_{1} \times N_{2}\right) & =N_{1} \operatorname{det}\left(N, N_{12}, N_{2}\right)+N_{2} \operatorname{det}\left(N, N_{1}, N_{12}\right) \\
& =N_{12} \operatorname{det}\left(N, N_{1}, N_{2}\right)+N \operatorname{det}\left(N_{12}, N_{2}, N_{1}\right)
\end{aligned}
$$

we read off the only way to solve (8), which is also illustrated by Fig. 9a:

$$
\frac{\lambda_{2}}{\lambda_{1}}=\frac{\operatorname{det}\left(N, N_{12}, N_{2}\right)}{\operatorname{det}\left(N, N_{1}, N_{12}\right)}, \quad \frac{\lambda}{\lambda_{12}}=\frac{\operatorname{det}\left(N, N_{1}, N_{2}\right)}{\operatorname{det}\left(N_{12}, N_{2}, N_{1}\right)} .
$$

These ratios of determinants equal the ratio of respective triangle areas, because the vertices which occur in each fraction are coplanar. Observe that all vertex triples have the same orientation. Apparently the choice of $\lambda(i, j)$ determines $\lambda(i+r, j+s)$ whenever $r+s \in 2 \mathbb{Z}$. A consistent assignment of $\lambda(i, j)$ 's is possible if circling a vertex leads to the same value of $\lambda$ again. In view of the comment on triangle areas, this is the condition expressed in the theorem.

Proposition 6. The fact that the triangle areas in a circular mesh contained in the unit sphere obey Equation (9) is a Möbius invariant.

Proof. This follows directly from the previous theorem, as isothermicity is a Möbius invariant.
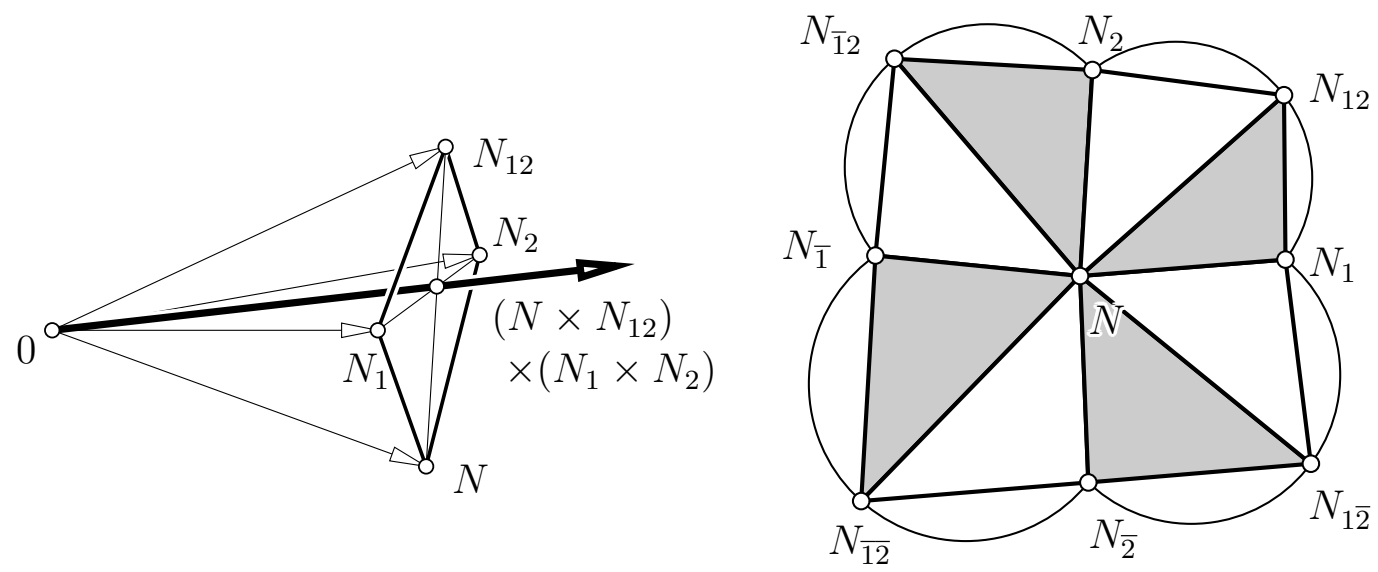

Figure 9: (a) Construction of normal vectors for the Lelieuvre formula. (b) For an isothermic mesh $N: \mathbb{Z}^{2} \rightarrow S^{2}$, the product of areas of the white triangles equals the product of the areas of the grey triangles. 


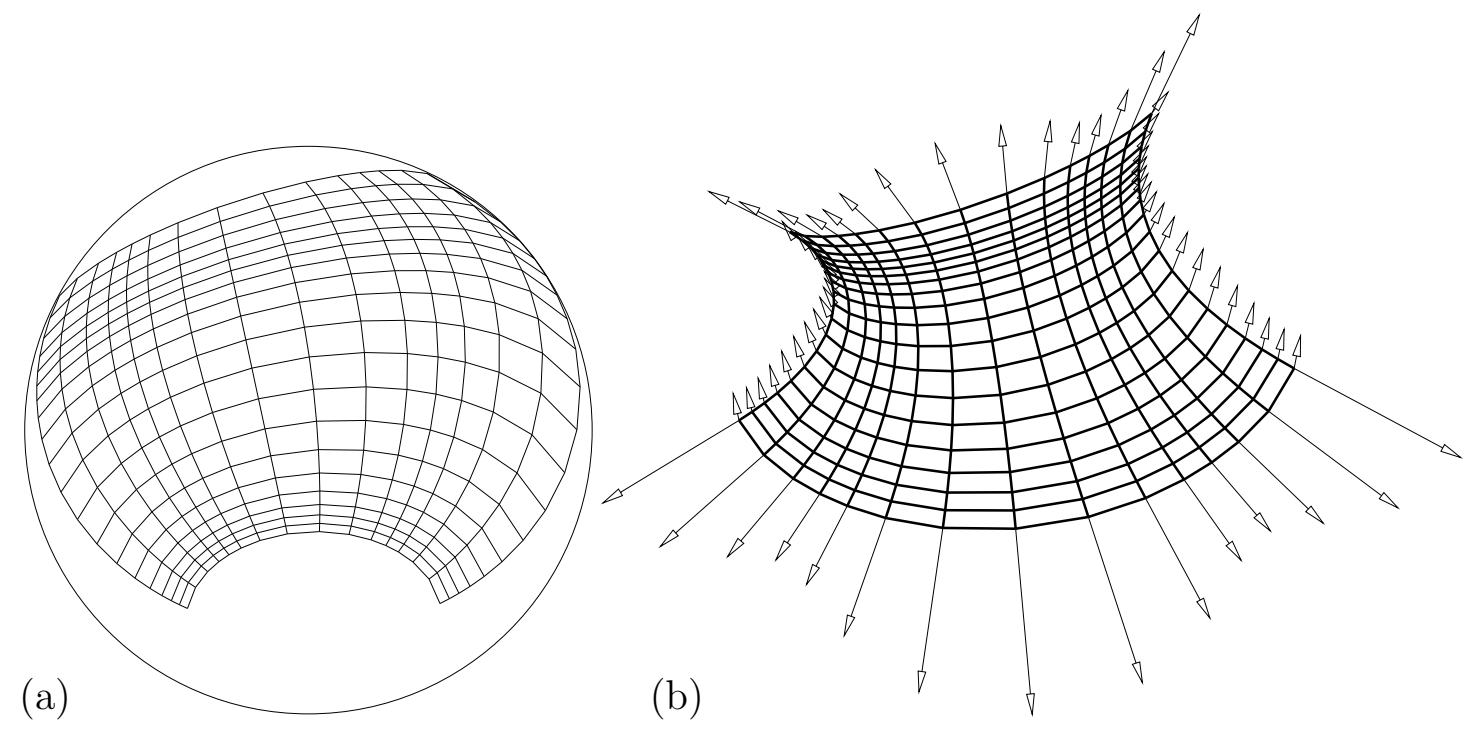

Figure 10: (a) An isothermic mesh $N: \mathbb{Z}^{2} \rightarrow S^{2}$. (b) The boundary edges of the discrete minimal surface $\mathscr{R}(N)$ (asymptotic mesh) serve as equilibrium forces for the discrete minimal surface $\mathscr{L}(N)$ (conical mesh).

\subsection{Statics}

All internal forces in a framework of rigid straight rods with flexible connections at either end act in the line of the rods. Each rod exerts a certain force onto a vertex it is connected to, and it exerts the negative of that force onto the vertex at its other end. For the framework to be in static equilibrium, for each vertex the sum of forces exerted onto it has to be zero.

We consider the edges of a quadrilateral mesh $V$ as a framework and ask the question if there exists a system of internal forces in the edges such that the mesh is in static equilibrium, if we allow external forces to be applied to boundary vertices.

Obviously a system of such forces can be arranged as the edges of a mesh $V^{\prime}$ which is reciprocal-parallel to $V$, and vice versa - the condition that the sum of forces in a vertex of $V$ is zero translates to the condition that these four vectors serve as the edges of a closed quadrilateral in $V^{\prime}$, and vice versa. Here a boundary vertex which has a number $e \leq 3$ vertices attached to it is endowed with $e$ external forces (see Fig. 8 and Fig. 2 for an illustration).

If $V^{\prime}, V^{\prime \prime}$ are two such collections of forces in the edges of $V$, then any linear combination $\lambda^{\prime} V^{\prime}+\lambda^{\prime \prime} V^{\prime \prime}$ is again a valid collection of forces. It follows that the vector space of equilibrium forces is naturally isomorphic to the vector space of meshes which are reciprocal-parallel to a given mesh.

These facts, which are elementary and well known, immediately show the following: 
Theorem 6. (i) A conical mesh $V$ possesses equilibrium forces if and only if its spherical image $\mathscr{S}(V)$ is isothermic. In particular, the discrete minimal surfaces of the form $\mathscr{L}(N)$ defined earlier have this property. The vector space of equilibrium forces has dimension 1.

(ii) An asymptotic mesh $V^{\prime}$ whose spherical image $N=\mathscr{S}\left(V^{\prime}\right)$ is circular, possesses equilibrium forces (and $N$ is isothermic). In case $V^{\prime}$ is finite with $n \times m$ faces, then the vector space of equilibrium forces has dimension $n+m$.

Proof. Statement (i) is Theorem 4. The dimension of the vector space of equilibrium forces follows from the fact that the mesh reciprocal-parallel to $V$ is unique up to scale.

As " " is a symmetric relation, statement (ii) follows from 5. Recall that with the polar dual $\widehat{N}$ of $N$, we have $V^{\prime} \sim \widehat{N}$. The set of equilibrium forces obviously

coincides with the set of Combescure transforms of $\widehat{N}$, whose dimension is $1 / 2$ times the number of boundary edges, i.e., $n+m$.

The previous theorem especially applies to discrete minimal surfaces in the shape of conical meshes. This discussion leads back to the original motivation for the construction of conical meshes in [11], which was applications in architecture connected to the existence of offset meshes and three-dimensional support structures consisting entirely of planar quadrilaterals. The existence of equilibrium forces is relevant for the construction of structures based on meshes and, more general, frameworks. Fig. 10 illustrates this fact.

\section{Conclusion and Future Research}

We have studied properties of circular and especially conical meshes which are connected with isothermicity and infinitesimal flexibility. It turns out that several properties of a quadrilateral mesh $N$ contained in the unit sphere are equivalent:

- isothermicity of $N$;

- the occurrence of a certain Möbius geometric Desargues configuration at each vertex of $N$;

- the infinitesimal flexibility of any conical mesh $V$ with spherical image $N$;

- Laguerre-isothermicity of $V$ (by definition);

- the fact that in the generic case any face of $V$ is co-spherical with its four diagonal neighbours;

- the existence of an asymptotic mesh $V^{\prime \prime}$ whose spherical image is $N$;

- a certain equality involving triangle areas within $N$; 
- the existence of a mesh $V^{\prime \prime}$ reciprocal-parallel to $V$.

Also the existence of a discrete minimal surface in the shape of a circular mesh whose discrete Weierstrass representation is $N$ (which is the topic of [4]) could be called a characterization of isothermicity. This last property led us to a construction of discrete minimal surfaces in the shape of conical meshes and to another one in the shape of asymptotic meshes (the last two being reciprocal parallels of each other). The construction of reciprocal-parallel meshes is finally used to to derive a result concerning the existence of equilibrium forces in conical meshes and discrete minimal surfaces.

Future work in this direction includes the investigation of discrete Laguerreminimal and relative-minimal surfaces, and a more thorough study of discrete analogues of objects of Möbius and Laguerre differential geometry. One candidate for such objects are the central spheres used in [1] as a basic tool of Laguerre surface theory.

\section{Acknowledgments}

The authors are grateful to A. Bobenko for his hospitality and many inspiring discussions. This research was supported by the Austrian Science Fund under grant No. S9206-N12.

\section{References}

[1] Blaschke, W. (1929), Vorlesungen über Differentialgeometrie, Vol. 3. Springer.

[2] Bobenko, A. (2006). personal communication.

[3] Bobenko, A., Hoffmann, T., and Springborn, B. A. (2006), Minimal surfaces from circle patterns: Geometry from combinatorics. Ann. of Math. 164:231264.

[4] Bobenko, A. and Pinkall, U. (1996), Discrete isothermic surfaces. J. Reine Angew. Math. 475:187-208.

[5] Bobenko, A. and Suris, Y. (2005), Discrete differential geometry. Consistency as integrability. preprint. http://arxiv.org/abs/math.DG/0504358.

[6] Bobenko, A. and Suris, Y. (2006), Isothermic surfaces in sphere geometries as Moutard nets. preprint, http://www.arxiv.org/abs/math.CO/0610434. 
[7] Bobenko, A. and Suris, Y. (2007), On Organizing Principles of discrete differential Geometry, Geometry of spheres. Russian Math. Surveys. to appear. http://arxiv.org/abs/math.DG/0608291.

[8] Cecil, T. (1992), Lie Sphere Geometry. Springer.

[9] Desbrun, M., Grinspun, E., and Schröder, P. (2005), Discrete Differential Geometry. SIGGRAPH Course Notes.

[10] Dyn, N. and Levin, D. (2002), Subdivision schemes in geometric modelling. Acta Numerica pp. 73-144.

[11] Liu, Y., Pottmann, H., Wallner, J., Yang, Y.-L., and Wang, W. (2006), Geometric Modeling with Conical Meshes and Developable Surfaces. ACM Trans. Graphics 25(3):681-689. Proc. SIGGRAPH 2006.

[12] Martin, R., de Pont, J., and Sharrock, T. J. (1986), Cyclide surfaces in computer aided design. In: J. A. Gregory (ed.): The mathematics of surfaces. Oxford: Clarendon Press, pp. 253-268.

[13] Pinkall, U. and Polthier, K. (1993), Computing discrete minimal surfaces and their conjugates. Experiment. Math. 2(1):15-36.

[14] Polthier, K. (2002), Polyhedral surfaces of constant mean curvature. Habilitationsschrift TU Berlin. http://page.mi.fu-berlin.de/polthier/articles/habil/ habilRev_Ch1_3_4.pdf.

[15] Polthier, K. (2005), Computational aspects of discrete minimal surfaces. In: Global theory of minimal surfaces, Vol. 2 of Clay Math. Proc. Amer. Math. Soc., pp. 65-111.

[16] Pottmann, H. and Peternell, M. (1998), Applications of Laguerre geometry in CAGD. Computer Aided Geom. Des. 15:165-186.

[17] Pottmann, H. and Wallner, J. (2007), The focal geometry of circular and conical meshes. Adv. Comp. Math. to appear, http://www.geometrie.tuwien. ac.at/wallner/focal.pdf.

[18] Sauer, R. (1933), Wackelige Kurvennetze bei einer infinitesimalen Flächenverbiegung. Math. Annalen 108:673-693.

[19] Sauer, R. (1970), Differenzengeometrie. Springer.

[20] Schief, W., Bobenko, A., and Hofmann, T. (2007), On the integrability of infinitesimal and finite deformations of polyhedral surfaces. In: A. Bobenko et al. (eds.): Proc. Oberwolfach Conference. Birkhäuser. to appear. 
[21] Wang, W., Wallner, J., and Liu, Y. (2006), An angle criterion for conical mesh vertices. Geometry Preprint 157, TU Wien. http://www.geometrie.tuwien.ac. at/ig/papers/tr157.pdf. 